$\xi=-$ 国

\title{
Analysis and Development of Restrictor for Parallel Twin Engine in FSAE Cars
}

\author{
M. Vignesh Kumar ${ }^{1 *}$, Sivanesan Murugesan ${ }^{2}$ \\ ${ }^{1}$ PG Scholar, Department Of Mechanical Engineering, Amrita School Of Engineering, Amrita Vishwa Vidyapeetham, Coimbatore, India. \\ ${ }^{2}$ Assistant Professor, Department Of Mechanical Engineering, Amrita School Of Engineering, Amrita Vishwa Vidyapeetham, \\ *Corresponding Author E-Mail:Vignesh.Leaf@Gmail.Com
}

\begin{abstract}
This research aims to the development of intake restrictor of a formula SAE car engine which is of 300cc parallel twin cylinder engine. In this paper we have considered different venturi designs which have different convergence and divergence angles. The main aim is to optimize the pressure and velocity of air which tends to offer better combustion reflects in performance. The parameters which are to be considered for design as well as analysis are mach number, intake velocity, mass flow rate, etc.., To perform this research we have chosen the Ansys fluent software tool and the analytical calculations were made for standard design. It is observed that the continuous variations in converging and diverging angles offers better results in both pressure and velocity characteristics of air entering for combustion.
\end{abstract}

Keywords: Restrictor, mass flow rate, optimize pressure and velocity.

\section{Introduction}

Formula SAE which is commonly called as FSAE, is a competition organized by Society of Automotive Engineers for students to exhibit their talents in design and development of a prototype of a car which should be under the regulations of SAE. This competition will be conducted every year in different parts of the world. In this competition the regulations are meant to be strictly followed. One of the rules is the usage of restrictor which should be of $20 \mathrm{~mm}$ diameter inlet which restricts the power of engine by the restriction of air flowing into engine.

The capacity of the engine which used in this car should be below $600 \mathrm{cc}$, which offers maximum power output of $110 \mathrm{hp}$, whether it can be a single cylinder or multi cylinder engine. Since at maximum rpm the engine requires more air which is restricted by the restrictor. But one advantage of restrictor is that it provides high velocity of air intake into engine. The flow of air attains a supersonic flow $(M=1)$. Here we work on the optimization of pressure and velocity of air which enhance the engine to offer better performance with minimum engine pull.

\section{Evolution Procedure}

The development procedure consists of five steps which includes manufacturing of restrictor. There is a linkage between each step which a small error reflects in the further steps. Usually there are two methods to restrict the flow of air / fluid. One is orifice and another one is converging diverging (Venturi) nozzle.

Table 1: Restrictor Characteristics

\begin{tabular}{|l|l|l|}
\hline PARAMETERS & ORIFICE & VENTURI \\
\hline Discharge Coefficient & 0.670 & 0.975 \\
\hline Pressure Drop & Medium & Low \\
\hline
\end{tabular}

\begin{tabular}{|l|l|l|}
\hline Viscosity & High & High \\
\hline Cost & Low & Medium \\
\hline Manufacturing method & Easy & Difficult \\
\hline Accuracy & 3 & 1 \\
\hline
\end{tabular}

As from the table differentiation, it is possible to conclude that venture-converging diverging nozzle offers better results of pressure drop results in high efficiency on the usage of restrictor of $20 \mathrm{~mm}$. By the analytical calculations the diameter of throttle for a $300 \mathrm{cc}$ parallel twin cylinder and the rpm of 11500 , is to be $30 \mathrm{~mm}$ and the outlet is to be changed to $25 \mathrm{~mm}$ so that it offers better velocity ratio. This will be diameter of the inlet and outlet of venturi - Restrictor.

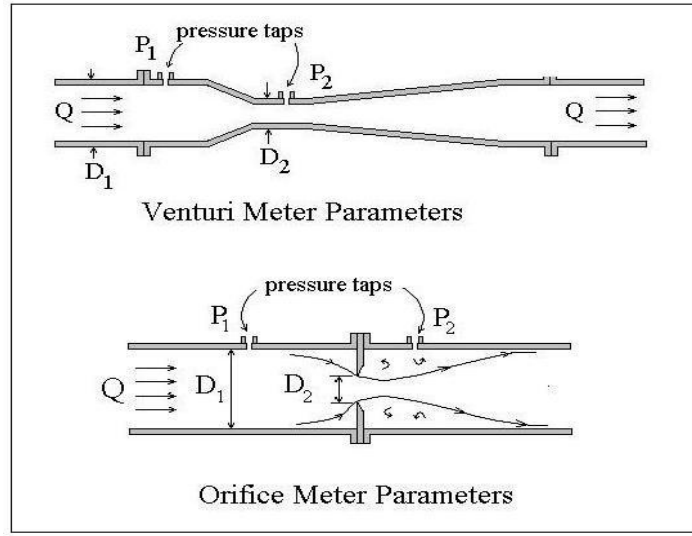

Table 2: Specifications of Engine

\begin{tabular}{|l|l|}
\hline Type of engine & Parallel twin \\
\hline Displacement & $300 \mathrm{cc}$ \\
\hline No of Cylinders & 2 \\
\hline Maximum Power & 37.73 Bhp @ 11500rpm \\
\hline Maximum torque & $26.5 \mathrm{Nm} @ 10000 \mathrm{rpm}$ \\
\hline Compression ratio & $12: 1$ \\
\hline Bore $*$ Stroke & $65 * 42.5 \mathrm{~mm}$ \\
\hline
\end{tabular}


\begin{tabular}{|l|l}
\hline Engine Description & 4 stroke, liquid cooled, 8 valves, DOHC
\end{tabular} Fuel system Electronic injection

\section{Analytical Calculations}

To find out the known and unknown values determines the flow conditions at the inlet and outlet. The obtained parameters offer better boundary conditions at the time of CFD analysis. Thus we have the known values like temperature of air at inlet which is ambient and the pressure is atmospheric. The known design values are the converging and diverging angle, which states the length of the venturi. The outlet can be of pressure, velocity, mass flow rate etc.., In order to perform analysis it is necessary to find out the mass flow rate at the condition of choked flow.

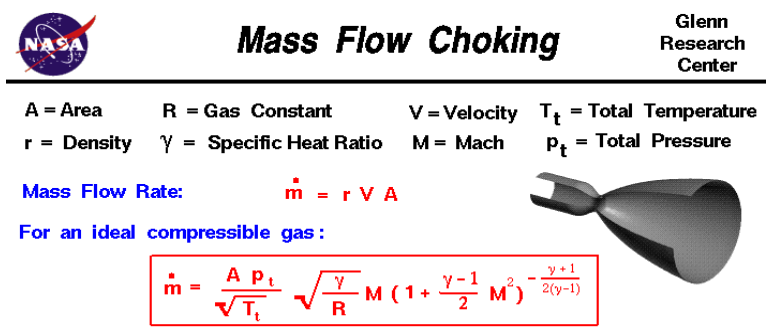

Mass Flow Rate is a maximum when $M=$ At these conditions, flow is choked.

$$
\dot{m}=\frac{A p_{t}}{\sqrt{T_{t}}} \sqrt{\frac{\gamma}{R}}\left(\frac{\gamma+1}{2}\right)^{-\frac{y+1}{2(y-1)}}
$$

Fig. 1: Mass flow choking condition

For 300cc parallel twin cylinder the mass flow rate can be calculated from the following values,

$\mathrm{P}=101325 \mathrm{~Pa}$

$\mathrm{A}=0.001256 \mathrm{~m}^{2}$

$\mathrm{R}=0.287 \mathrm{KJ} / \mathrm{Kg}-\mathrm{K}$

$\gamma=1.4$

$\mathrm{T}=300 \mathrm{~K}$

Mass flow rate $=0.0706 \mathrm{Kg} / \mathrm{s}($ choked flow $\mathrm{M}=1)$

Throttle diameter $=\frac{\sqrt{154.2 n l N d^{2}}}{67547}$

Where

$\mathrm{N}=$ Revolutions per minute $\mathrm{d}=$ bore diameter in inches

$l=$ stroke length in inches

$\mathrm{n}=$ number of cylinders

Throttle diameter $=\mathbf{2 5} \mathbf{~ m m}$ (300cc twin cylinder)

The dimensional parameters and the boundary values for the design of restrictor is mentioned in the below table

Table 3: Dimensional Values for Restrictor

\begin{tabular}{|l|l|}
\hline PARAMETERS & VALUES \\
\hline Inlet diameter & $30 \mathrm{~mm}$ \\
\hline Restrictor diameter & $20 \mathrm{~mm}$ \\
\hline Throttle diameter & $25 \mathrm{~mm}$ \\
\hline Convergence angle & $10-20$ degrees \\
\hline Divergence angle & $5-10$ degrees \\
\hline Length of restrictor & $150 \mathrm{~mm}$ \\
\hline Inlet pressure & $1.01 \mathrm{bar}$ \\
\hline Inlet temperature & $298 \mathrm{~K}$ \\
\hline Mass flow rate & $0.0703 \mathrm{~kg} / \mathrm{s}$ \\
\hline Viscosity of air & $1.225 \mathrm{Kg} / \mathrm{m}^{3}$ \\
\hline Density of air & $1.81 * 10^{-5} \mathrm{~Pa}$ \\
\hline
\end{tabular}

\section{Dynamics of Fluid}

Most important parameter which determines the flow of fluid through a restrictor is Mach number, which tends to determine whether the flow is subsonic $M<1$ or supersonics $M<1$. The Mach number can be calculated from the flow velocity and the speed of sound $\mathrm{M}=\mathrm{V} / \mathrm{C}$, where $\mathrm{V}=$ flow velocity, $\mathrm{C}=$ speed of sound. Another reason to consider Mach number is to determine the condition of density of air while experiencing flow through restrictor.

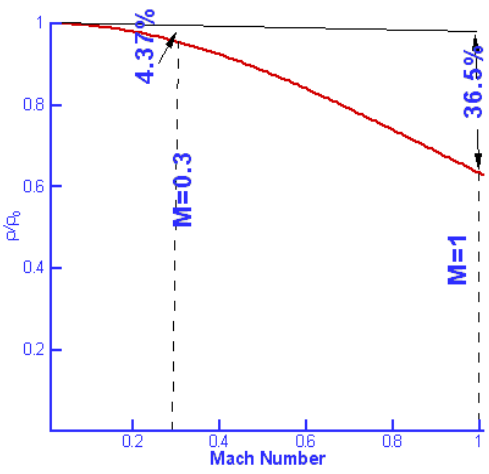

Fig. 2: Mach number vs density

The converging diverging nozzle reduces the density of air during flow which affects the power of engine when the engine attain is maximum pull which tends to occurs at 7000rpm and more. This tends to create suffocation of air inside the engine and slower the engine at higher rpm. It is necessary to recover higher pressure outlet from the restrictor.

Qualitive variation of $\mathrm{Cd}$ with Mach number

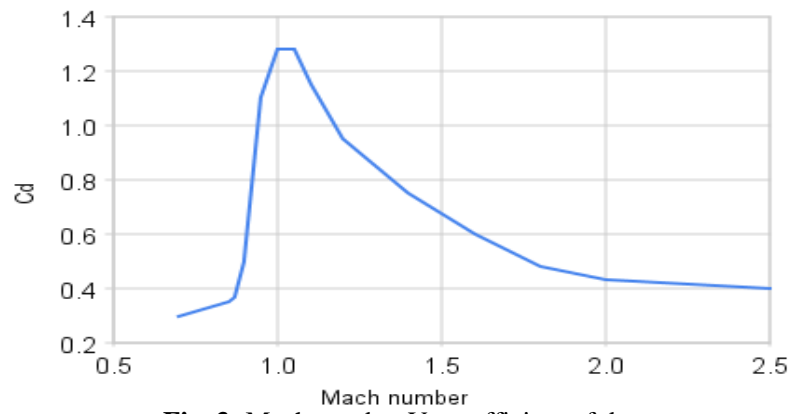

Fig. 3: Mach number Vs coefficient of drag

\section{Fluent Analysis}

It is necessary to choose right software for the flow analysis and also the modeling software. Here we have used ANSYS FLUENT for creating geometry and also to perform CFD analysis. Hence it offers better parameters which can be used in analysis results in better mass flow rate of air. In order to compare mass flow rate corresponding to different converging and diverging angles are defined and respective iterations have been done to conclude the minimum pressure drop in the restrictor.

\section{Manufacturing Process}

Once the design and analysis part have been completed it is necessary to convert into product. In order to attain a prototype it is necessary to implement better manufacturing steps. Two methods can be used either rapid prototyping or sheet metal process. Rapid prototyping is most commonly used since there is no need any external finishing operations like in sheet metal and offers more accuracy in converging and diverging angles and also the diameter of inlet and outlet.

\section{Analysis \& Iterations}

Once we have gathered all values required for analysis, it is necessary perform segregation to so that it is possible to get clear idea of analysis for the corresponding iterations. The initial values for the analysis are to be assumed based on the operation of venturi.

Inlet: pressure inlet $-101325 \mathrm{pa}$

Outlet: Mass flow rate $-0.0703 \mathrm{Kg} / \mathrm{s}$ 
The number of iterations to be performed and the corresponding values of iterations are tabulated below:

Table 4: Iterative Values for Analysis

\begin{tabular}{|l|l|l|}
\hline ITERATION & CONVERGING ANGLE & DIVERGING ANGLE \\
\hline 1 & 10 & 5 \\
\hline 2 & 12 & 6 \\
\hline 3 & 14 & 7 \\
\hline 4 & 16 & 8 \\
\hline 5 & 18 & 9 \\
\hline 6 & 20 & 10 \\
\hline
\end{tabular}

The flow analysis is performed using ANSYS FLUENT software and the modeling of restrictor is done by the same. The variations in the pressure drop based on the iterations are tabulated below:

Table 5: Results for the Iterative Values after Analysis

\begin{tabular}{|l|l|l|}
\hline ITERATION & PRESSURE VALUES $(\mathrm{Pa})$ & VELOCITY $(\mathrm{m} / \mathrm{s})$ \\
\hline 1 & 19523 & 80.5 \\
\hline 2 & 19700 & 75.3 \\
\hline 3 & 19924 & 70 \\
\hline 4 & 20654 & 73 \\
\hline 5 & 20600 & 72.5 \\
\hline 6 & 24719 & 74 \\
\hline
\end{tabular}

The results obtained from the contour plots are converted into graphs. The graphs are plotted for both pressure and velocity variations which show the results of better iterative values which can be implemented in design of restrictor.

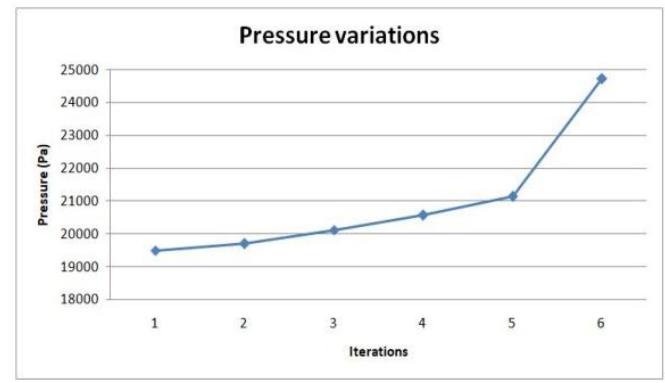

Fig. 4: Pressure plot for the iterative results

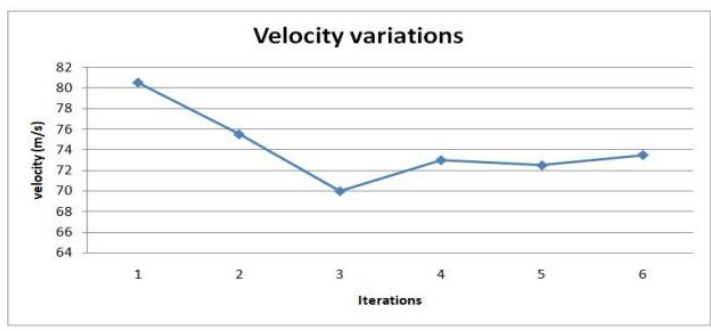

Fig. 5: Velocity plot for the iterative results

After completion of analysis based on the values specified it is necessary to get into a result that which of the iteration and its corresponding values tends to offer better efficiency. The convergence and divergence angle variations tend to affect the streamline flow of air and also the pressure drop. Among those iterations divergence angle of degrees does not affect both the parameters. Some other variations of parameters for the final design are follows,

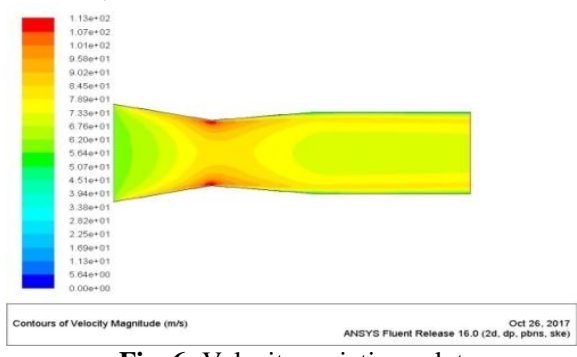

Fig. 6: Velocity variations plot

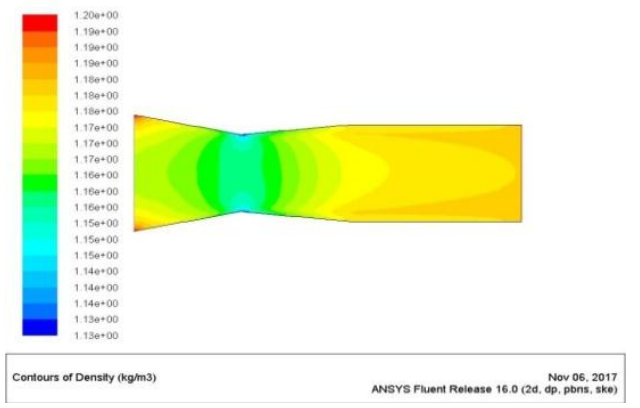

Fig. 7: Density variations plot

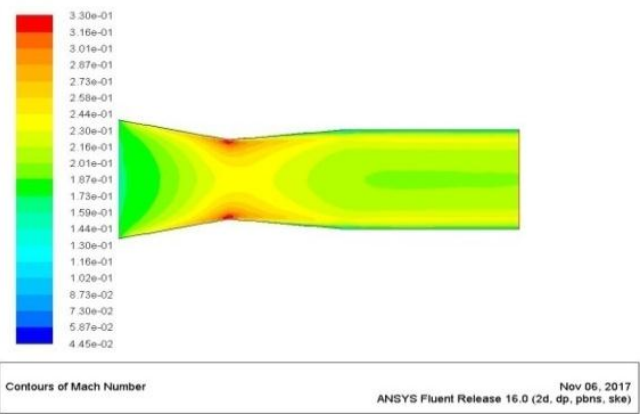

Fig. 8: Mach number variations plot

\section{Conclusion}

In this paper, venturi type restrictor is used in order to provide better mass flow rate of engine. Since all other teams were interested to drawn out maximum power from engine it is necessary to spend more research in optimization and at the same time it should provide better mass flow rate. By usage of venturi it is possible to attain the mass flow rate of $0.0703 \mathrm{Kg} / \mathrm{s}$ at the maximum condition irrespective of turbulence and no losses due to friction.

From all the analysis process it is possible to conclude that the converging angle and diverging angle of 10 and 5 degrees offers better pressure recovery because these angles does not have intricate structure which causes higher pressure drop and also offers better laminar flow of air. To complete this research paper Computational fluid dynamics offers better facilities in all aspects.

\section{References}

[1] Aadepu S, Prasanth ISNVR \& Naik JM, "Design of intake manifold of IC engines with improved volumetric efficiency", International Journal \& Magazine of Engineering, Technology, Management And Research, Vol.1, No.6, (2014), pp.26-33.

[2] Thirumalini S, Lakshmikanthan C \& Dhandapani S, "CFD Modeling for parametric investigation of flow through the inlet valve of a four-stroke engine", International journal of Applied Engineering research, Research India publications, (2009).

[3] Singhal A \& Parveen M, "Air flow optimization via venturi type air restrictor", World Congress of Engineering, (2013).

[4] Shinde PA, "Research and optimization of intake restrictor of a formula SAE car engine", International Journal of Scientific and Research publications, (2014).

[5] Raj S, Kr. Singh A, Srivastava T \& Vibhanshu V, "Analysis of Air Intake for Formula SAE Vehicle", Vth International Symposium on "Fusion of Science \& Technology", (2016).

[6] Madhu NT, et.al, "Design and simulation of a fluid flow in paper based micro fluidics Platforms", International conference on Advanced materials, (2016).

[7] well MC \& Horkheimer D, "Improvement of intake restrictor performance for a formula SAE car thorough $1 \mathrm{D} \&$ coupled $1 \mathrm{D} / 2 \mathrm{D}$ analysis methods" SAE technical Papers, 2006.

[8] Srikrishnan AR, "Validation of CFD software: A study of issues specific to simulation of higher mach number flows", International conference on CAE, IIT Chennai, (2013). 
[9] Shelagowski LM \& Mahank TA, "CFR formula SAE car Intake restrictor Design and performance", American Society of Engineering Education, (2015).

[10] Waghmare SN, Karekar NS, Karande PP \& Pandhare SB, "Design and analysis of intake system of formula SAE car", International journal of Engineering technology, Management and applied sciences, (2016).

[11] Haridas M, Gopal G, Ramesh A \& Katta RK, "Modelling and simulation of single and multi-pass flow forming to investigate the influence of process parameters on part accuracy", International Journal of Manufacturing Research, Vol.11, No.3,(2016), pp.274289.

[12] Plank MR, "Engine optimization and performance characteristics for a Formula SAE race Car", University of Southern Queensland, Queensland, (2015).

[13] Ceviz M, "Intake plenum volume and its Influence on the engine", Science Direct, (2006)

[14] Sayed A, "Air flow optimization through an intake system for a single cylinder FSAE car", International journal of Engineering and Technology, (2017).

[15] http://www.hipermath.com/math_center/racing_calculations.

[16] http://www.msubbu.in/ln/fm/UnitIII/VenturiOrifice.html 